\section{BRONCHITIS-EXAZERBATION}

\section{Was die Sputumfarbe wirklich verrät}

Die Farbe des Auswurfs kann ein Indikator dafür sein, ob ein Patient mit akuter Exazerbation einer chronischen Bronchitis (AECB) von einem Antibiotikum profitieren würde. Spanische Ärzte haben dies anhand von sechs Studien untersucht, in denen verschiedene Antibiotika bei AECB verglichen worden waren. In 4003 Fällen existierten Angaben zur Farbe des Sputums. Bakterien, mehrheitlich $\mathrm{H}$. influenzae, konnten in 1898 Fällen $(46,4 \%)$ isoliert werden. 58,9\% der grünen und $45,5 \%$ der gelben Sputumproben enthielten potenziell pathogene
Mikroorganismen, aber nur $18,4 \%$ der klaren/weißen Proben. Verglichen mit weißem Sputum detektierte grünes oder gelbes Sputum Bakterien mit einer Sensitivität von $94,7 \%$. Die Spezifität betrug allerdings nur $15,0 \%$. Der Farbencheck produziert also auch viele falsch-positive Befunde. Nicht verfärbtes Sputum ist der Studie zufolge jedoch ein guter Prädiktor für einen negativen Kulturbefund. Hier kann man, so die Autoren, wohl auf Antibiotika verzichten.

ERJ 2011; published online Okt. 27, 2011 DOI:10.1183/09031936.00042111

\section{KANN DAS DIE KINDLICHE ENTWICKLUNG STÖREN?} \section{„Eisenmilch“ im Fläschchen}

Bei ausschließlich gestillten Säuglingen gehen im Alter von etwa vier bis sechs Monaten die Eisenspeicher zur Neige; ab diesem Zeitpunkt wird eine ausreichend eisenhaltige Ernährung empfohlen, um evtl. Verzögerungen in der neurologischen Entwicklung zu vermeiden. Die Ergänzung über eisenangereichertes Milchpulver hat aber offenbar eine Kehrseite: So schnitten Kinder, die ab dem sechsten Monat bis zum Alter von einem Jahr Folgemilch mit einem Eisengehalt von durchschnittlich $12,7 \mathrm{mg} / \mathrm{l} \mathrm{er-}$

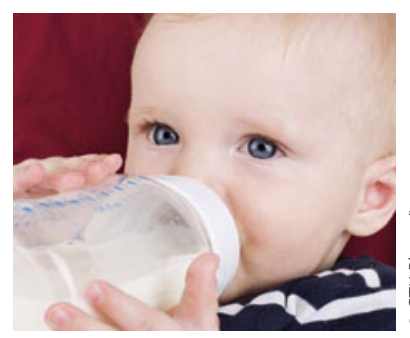

Nicht zu viel des Guten! halten hatten, in kognitiven und visuomotorischen Tests nach zehn Jahren zum Teil deutlich schlechter ab als Kinder, die eisenärmere Milch (2,3 mg/l) genuckelt hatten. Allerdings war die „Eisenmilch“ der Entwicklung nur dann abträglich, wenn ursprünglich kein Eisenmangel vorgelegen hatte. Arch Pediatr Adolesc Med 2011; published online Nov. 7; doi:10.1001/archpediatrics.2011.197

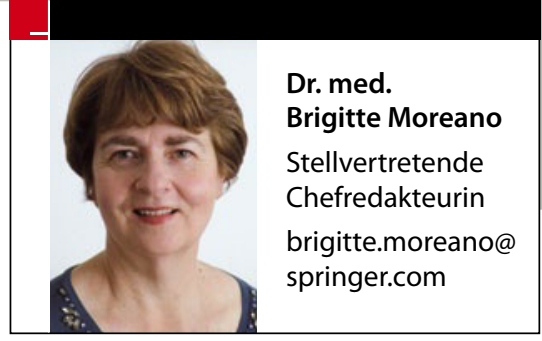

OTITIS BEI KINDERN

Kaugummi fürs Ohr

Kauen Kinder Kaugummis mit dem Süßstoff Xylit, können sie dadurch jede vierte Otitis media vermeiden. Die bakterienhemmende Eigenschaft von Xylit wirkt sich bis zum Mittelohr aus, ergab eine CochraneAnalyse von drei placebokontrollierten finnischen Studien. In diesen erhielten über 1800 Kinder unter zwölf Jahren entweder Kaugummis, Lutschtabletten oder einen Sirup mit dem Süßstoff in einer Tagesdosis von 8-10 g - oder aber solche Präparate ohne Xylit. Im Schnitt dauerten die Studien zwei bis drei Monate. Insgesamt kam es in dieser Zeit zu 473 Mittelohrentzündungen Die Otitis media war in den Gruppen mit Xylitpräparaten deutlich seltener als in den Kontrollgruppen. Am effektivsten schien der Kaugummi: Mit Xylit (vs. Placebo) war die Otitis-media-Rate um $41 \%$ reduziert, mit Lutschtabletten und Sirup nur um 20\%.

Cochrane Review 2011/11, Epup 9.11.2011

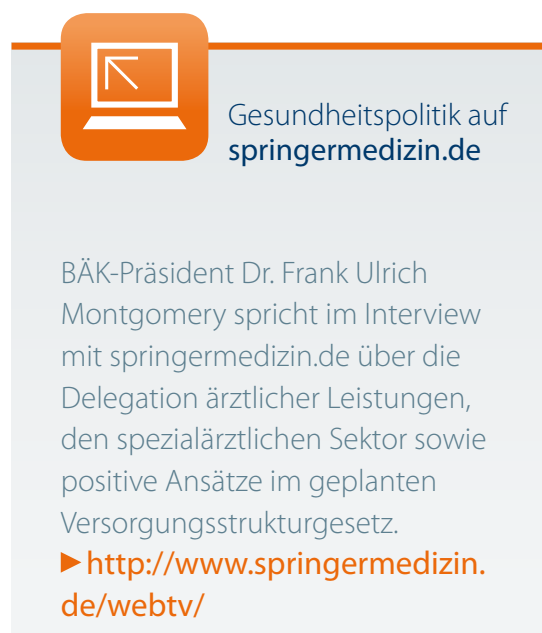

\title{
PROTEKTIVER EFFEKT
}

\section{Ballaststoffe gegen Darmkrebs}

Wer sich ballaststoffreich ernährt, reduziert sein Risiko für ein kolorektales Karzinom, so das Ergebnis einer Metaanalyse von 25 prospektiven Studien mit nahezu zwei Millionen Teilnehmern. Pro $10 \mathrm{~g}$ aufgenommenen Ballaststoffen sinkt das Risiko für Darmkrebs um 10\%. Wer $90 \mathrm{~g}$ Vollkorn täg- lich zu sich nimmt, reduziert sein Risiko um $20 \%$. Einen besonders großen Schutzeffekt wiesen Cerealien und Vollkornprodukte auf. Ob auch Obst und Gemüse das Risiko senken, konnte in dieser Analyse nicht gezeigt werden.

BMJ 2011;343:d6617 doi: 10.1136/bmj.d6617 